\title{
Depression, anxiety, and fear of COVID-19 in patients with multiple sclerosis in pandemic era: a cross-sectional study
}

\author{
Mohammad Alirezaei $^{1} \cdot$ Sharareh Eskandarieh ${ }^{1} \cdot$ Mohammad Ali Sahraian $^{1} \cdot$ Abdorreza Naser Moghadasi $^{1}$ (I)
}

Received: 12 April 2021 / Accepted: 10 September 2021 / Published online: 23 September 2021

(c) Fondazione Società Italiana di Neurologia 2021

\begin{abstract}
Background Depression and anxiety are the two important factors determining quality of life of patients with multiple sclerosis (PWMS). In COVID-19 pandemic era, several factors can provoke mental issues of people and patients. In this cross-sectional study, we aim to estimate the new prevalence of anxious and depressive symptoms and their relating factors in PWMS.

Methods In this cross-sectional study, we include PWMS who are recruited in the MS clinic of Sina Hospital, Tehran, and are joined in our channel of Telegram media. A self-designed online questionnaire consisted of 4 parts handed out between patients: demographic and clinical data, Beck depression inventory, Beck anxiety inventory, and Fear of COVID-19 Scale. Univariate and multiple logistic regression analyses were performed to find the relating factors of expression of depressive and anxious symptoms in PWMS.

Results Of a total of 282 participants with the mean age of 35.66 (30.75-40) years, had been suffering from multiple sclerosis for 7.36 (3-10) years, $81.7 \%$ were women and $69.1 \%$ classified as relapsing-remitting MS. Mean score of BDI was $17.13 \pm 11.51$ which is classified as minimal-moderate depressive symptoms. $48.6 \%$ of patients did not express depressive symptoms $(\mathrm{BDI}-\mathrm{II} \leq 14)$ and the others reported some degrees of depression. In the univariate analysis employment $(p=0.015)$, marital status $(p=0.022)$, level of education $(p=0.004)$, number of hospitalization due to MS attacks $(p=0.048)$, and fear of COVID-19 ( $p \leq 0.0001)$ associated significantly with presence of depressive symptoms. After entering these factors in a binary logistic regression model, level of education $(p=0.019)$, marital status $(p=0.044)$, number of hospital admissions due to MS relapses $(\beta=1.10, p=0.02)$, and fear of COVID-19 $(\beta=1.07, p \leq 0001)$ remained significant as relating factors. Mean score of the anxiety calculated $14.54 \pm 9.75$ and just $3.2 \%$ of patients had severe anxiety. Employment $(p=0.045)$, EDSS score $(p=0.004)$, and fear of COVID-19 $(p \leq 0.0001)$ reported relating to anxious symptoms significantly in the univariate analysis. After entering in the logistic regression analysis, EDSS $(\beta=1.30, p=0.001)$ and fear of COVID-19 $(\beta=1.13, p \leq 0.0001)$ remained as significant relating factors of anxious symptoms.

Conclusion The overall prevalence of depressive symptoms in PWMS in our MS clinic is $51.4 \%$ which is obviously higher than other world's centers which could be due to fear of COVID-19. In addition to fear of COVID-19, presence of depressive symptoms in PWMS is related significantly with level of education, number of hospital admissions due to MS relapses, and marital status. Other side, the patients classified as suffering from anxious symptoms had more severe problems on fear of COVID-19. But it is recommended for future studies to compare patients score in the COVID-19 era with their score before this pandemic.
\end{abstract}

Keywords Depression $\cdot$ Anxiety $\cdot$ Fear of COVID-19 $\cdot$ Multiple sclerosis

\section{Introduction}

Abdorreza Naser Moghadasi

abdorrezamoghadasi@gmail.com

1 Multiple Sclerosis Research Center, Neuroscience Institute,

Tehran University of Medical Sciences, Tehran, Iran

One of the recent most important health issues concerning the world health is the spread of COVID-19 pandemic, the first case of which was reported on December 2019 in Wuhan, China [1]. This betacoronavirus with the 
single-stranded, enveloped RNA, belonging to the Coronaviridae family, is the seventh type of coronavirus in humankind [2].

Beyond the physical effects and neurological involvements, every newly defined pandemic can potentially lead to psychological effects in all members of a society. Usually, at the beginning of the emergence of any new disease, on the one hand, due to the lack of specific, efficient, and effective treatments, and on the other hand, due to the successive questioning of existing information about every aspect of this disease, people would be prone to psychological disease. Furthermore, large-scale involvement in the disease may lead to adoption of strict policies such as public quarantine resulting in the reduction of the level of social communication, increase in domestic violence, reduction in access to public recreation, and suspension of payments (i.e., people may lose their revenue sources) [3].

The most common psychiatric problems with the highest DALY (disability-adjusted life years) in Western societies are depressive and anxiety disorders. For instance, in the USA, between 5.1 and $11.9 \%$ of the population suffer from generalized anxiety disorder [4-7], and the lifetime prevalence of a minor depression episode and a major depression disorder is $9-10 \%$ and $17 \%$, respectively [5, 8]. In the last decades, the severity and prevalence of depressive disorders have promoted from subsyndromal and minor depression to major depression disorder (MDD) $[9,10]$. The point prevalence of MDD has more than doubled from 1991 to 2001 in two nationally representative surveys in the USA, and the prevalence of depressive disorders in 2011 has reached about $18 \%$ in the developed countries [11].

Some studies have reported that this pandemic is leading to different health problems such as anxiety, insomnia, anger, depressive symptoms, denial, and fear globally [12] and had a mild stressful impact on people [13].

In addition to the general population, these two mood disorders have a special role in the course of other chronic diseases, including multiple sclerosis. According to recent review articles, the most common comorbidity associated with multiple sclerosis is mood disorders, particularly depression [14], where up to $30-50 \%$ of patients suffer from depression and up to $22.1 \%$ of patients suffer from anxiety $[15,16]$. In multiple sclerosis population, the level of anxiety has increased compared to the recent year and before the pandemic. The individuals' most concerns were the possibility of the involvement and death of their acquaintances, as well as the lack of specific treatment for this epidemic $[17,18]$.

Various studies have shown the impacts of depression on the patients' quality of life. In this regard, a global review article published in 2020 examined factors associating health-related quality of life in MS patients. It was revealed that age, depression, and disease duration had the greatest impact, but fatigue and expanded disability status scale (EDSS) had the less effect [17, 19]. Various studies have also shown the effect of anxiety and stress on the onset of MS and its relapses [20]. Besides these physical effects, recent efforts are made to evaluate the relation between depression and mood disorders, and invisible complications of MS, like fatigue and cognitive function [21].

In addition to depression and anxiety, fear of COVID19 in people and patients is another psychosocial effect of this pandemic, knowing the severity of which is essential in recognizing whether preventive and lockdown strategies are required [22]. Furthermore, it affects the medication seek in patients with chronic or acute diseases and may result in psychological complications and life dissatisfaction [23].

In this study, we aimed at estimating the prevalence of depression and anxiety in patients with multiple sclerosis (PWMS) in the COVID-19 outbreak and evaluating the potential relating factors which may predict their prevalence.

\section{Materials and methods}

\section{Participants}

This study was conducted in a cross-sectional manner in PWMS, including the patients who recruited in the MS clinic of Sina Hospital, Tehran, Iran, in August and October 2020. All patients were definitely diagnosed with MS or clinically isolated syndrome by a neurologist based on the McDonald's diagnostic criteria revised in 2017 [24]. In order to provide remote services or consultations and avoid unnecessary visits, all patients were invited to join in a channel on the Telegram social media. This study was approved by the ethical committee of Tehran University of Medical Sciences (IR.TUMS.SINAHOSPITAL.REC.1399.065).

A four-part self-designed questionnaire was prepared and administered on-line for data gathering by a general practitioner. After explaining the aim and design of the study, the patients were asked to answer the questions within the coming 3 weeks, unless they had a relapse and steroid therapy within the last month. Out of 453 members of the channel, 282 patients accepted to participate in the survey and filled all the parts completely. The first part was about demographic and clinical features of the participants. Second and third parts were the Beck depression inventory [25] and Beck anxiety inventory [26] consisting of 21 multiplechoice questions with a total score of 63 . Patients with a score of 15 and 11 or higher were classified as presence of depressive and anxious symptoms, respectively. The last part was assigned to evaluate the severity of fear of COVID19 in the participants. A 7-item questionnaire validated on 
Iranian participants with the overall score of the summed-up items, ranged from 7 to 35 indicated the severity of the fear of COVID-19 [27].

\section{Variable definition}

Ongoing and past medical condition of patients were asked with clear questions in first part of the questionnaire, including type of MS, disease duration, treatment duration, medication, number of hospital admissions, EDSS score, past psychiatric history, and history of COVID-19 infection. Disease duration and treatment duration were considered as the years passed from physician diagnosis and the first time the patients have been using medications regularly. For estimating the frequency of relapses, we just included the attacks leading to hospitalizations and entered them as a quantitative variable in the analysis.

Past psychiatric history of patients was asked by whether they have ever been prescribed a medication by a physician for psychological issues. All data were rechecked according to the patients' medical records.

\section{Statistical analyses}

Descriptive analysis was reported as number (percentage) and mean (first and third quartiles) for qualitative variables and quantitative variables with abnormal distributions. Normality of the study population was examined by Kolmogorov-Smirnov test.

First, different variables across depression and anxiety were examined through chi-square, $U$ Mann-Whitney, and Kruskal-Wallis test. Finally, age, gender, and past history of psychological diseases were fixed in the univariate logistic regression model as potential confounders and all other significant factors revealed in the first step were examined in an enter model. All $p$ values were considered as 2-tailed with a statistical significance of $p \leq 0.05$. SPSS statistical software version 23 was used for statistical analyses.

\section{Results}

Of a total of 282 participants ( $81.7 \%$ female) with a mean age of 35.66 (30.75-40) years, 208 patients $(73.7 \%)$ had college education while others did not have. Most (69.1\%) of them were suffering from relapsing-remitting multiple sclerosis and only $7.1 \%$ had been diagnosed as CIS. They have experienced $2.23(0-2)$ hospital admissions due to MS attacks in a 7.36 (3-10) years of disease duration. Of 26 participants reported as recent cases involved with COVID19 , only one patient was admitted in hospital for supportive therapy. BDI and BAI indicated that 145 (51.4\%) and 171
$(60.2 \%)$ of patients scored higher than 14 and 10; they were classified as presence of depressive and anxious symptoms, respectively. See Table 1 for a comprehensive description of the study population.

Among all demographic features and clinical factors in Table 1 , education ( $p$ value $=0.004)$, employment $(p$ value $=0.015)$, marital status $(p$ value $=0.022)$, number of hospital admission due to MS attacks ( $p$ value $=0.048)$, and severity of fear of COVID-19 ( $p$ value $\leq 0.0001$ ) had significant differences in the presence of depressive symptoms (Table 2).

For prediction of the prevalence of depressive symptoms in PWMS, level of education $(p$ value $=0.019)$, marital

Table 1 Description of the study sample

\begin{tabular}{ll}
\hline Characteristics & Mean or number \\
\hline Age** & $35.66(30.75-40)$ \\
Gender (female)* & $232(81.7 \%)$ \\
Education* & \\
$\quad$ Middle school degree & $10(3.5 \%)$ \\
$\quad$ High school diploma & $64(22.5 \%)$ \\
$\quad$ Associate/bachelor degree & $149(52.5 \%)$ \\
$\quad$ Graduate degree & $59(20.8 \%)$ \\
Marital status* & \\
$\quad$ Single & $93(32.7 \%)$ \\
$\quad$ Married & $155(54.6 \%)$ \\
Divorced & $34(12 \%)$ \\
Employment (yes)* & $108(38 \%)$ \\
MS type* & \\
CIS & $19(6.7 \%)$ \\
$\quad$ RRMS & $197(69.4 \%)$ \\
PPMS & $22(7.7 \%)$ \\
SPMS & $46(16.2 \%)$ \\
Disease duration** & $7.36(3-10)$ \\
Treatment duration** & $6.21(3-9)$ \\
Drug* &
\end{tabular}

*Qualitative variables reported as number (percent)

**Quantitative variables with abnormal distribution reported as mean (first and third quartiles)

$C I S$, clinically isolated syndrome; RRMS, relapsing-remitting multiple sclerosis; PPMS, primary progressive multiple sclerosis; SPMS, secondary progressive multiple sclerosis; EDSS, expanded disability status scale; $B D I$, Beck depression inventory; $B A I$, Beck anxiety inventory. 
Table 2 Qualitative and quantitative variables across expression of depressive symptoms

\begin{tabular}{|c|c|c|c|}
\hline & \multicolumn{2}{|c|}{ Expression of depressive symptoms } & \multirow[t]{2}{*}{$p$ value } \\
\hline & Yes & No & \\
\hline Age & $35.10(29-40)$ & $36.26(32-41)$ & 0.310 \\
\hline Gender & & & 0.247 \\
\hline Male & $22(15.2 \%)$ & $28(20.4 \%)$ & \\
\hline Female & $123(84.8 \%)$ & $109(79.6 \%)$ & \\
\hline Education & & & 0.004 \\
\hline Middle school degree & $8(5.5 \%)$ & $2(1.5 \%)$ & \\
\hline High school diploma & $43(29.7 \%)$ & $21(15.3 \%)$ & \\
\hline Associate/bachelor degree & $69(47.6 \%)$ & $80(58.4 \%)$ & \\
\hline Graduate degree & $25(17.2 \%)$ & $34(24.8 \%)$ & \\
\hline Occupational status & & & 0.015 \\
\hline Employed & $46(31.7 \%)$ & $62(45.9 \%)$ & \\
\hline Unemployed & $99(68.3 \%)$ & $73(54.1 \%)$ & \\
\hline Marital status & & & 0.022 \\
\hline Single & $44(30.3 \%)$ & $49(35.8 \%)$ & \\
\hline Married & $76(52.4 \%)$ & $79(57.7 \%)$ & \\
\hline Divorced & $25(17.2 \%)$ & $9(6.6 \%)$ & \\
\hline MS type & & & 0.547 \\
\hline CIS & $9(6.2 \%)$ & $10(7.3 \%)$ & \\
\hline RRMS & $97(66.9 \%)$ & $98(71.5 \%)$ & \\
\hline PPMS & $11(7.6 \%)$ & $11(8.0 \%)$ & \\
\hline SPMS & $28(19.3 \%)$ & $18(13.1 \%)$ & \\
\hline Disease duration & $7.31(3.5-10)$ & $7.41(3-10)$ & 0.930 \\
\hline Treatment duration & $6.04(3-7.5)$ & $6.4(3-9)$ & 0.513 \\
\hline Hospital admission due to MS attacks & $2.64(1-3)$ & $1.79(0-2)$ & 0.048 \\
\hline EDSS & $2.97(1.5-4.5)$ & $2.78(1.5-4)$ & 0.335 \\
\hline Drug type & & & 0.080 \\
\hline Immunomodulatory & $75(51.7 \%)$ & $85(62.0 \%)$ & \\
\hline Immunosuppressive & $70(48.3 \%)$ & $52(38.0 \%)$ & \\
\hline Past psychiatric history & & & 0.064 \\
\hline Yes & $35(24.1 \%)$ & $21(15.3 \%)$ & \\
\hline No & $110(75.9 \%)$ & $116(84.7 \%)$ & \\
\hline COVID-19 infection & & & 0.573 \\
\hline Yes & $12(8.3 \%)$ & $14(10.2 \%)$ & \\
\hline No & $133(91.7 \%)$ & $123(89.8 \%)$ & \\
\hline Fear of COVID-19 & $18.93(14-24)$ & $15.88(11.5-19)$ & $\leq 0.0001$ \\
\hline
\end{tabular}

$C I S$, clinically isolated syndrome; $R R M S$, relapsing-remitting multiple sclerosis; $P P M S$, primary progressive multiple sclerosis; SPMS, secondary progressive multiple sclerosis; EDSS, expanded disability status scale; $B D I$, Beck depression inventory; $B A I$, Beck anxiety inventory. status ( $p$ value $=0.44$ ), hospital admission due to MS attacks ( $p$ value $=0.02)$, and fear of COVID-19 $(p$ value $<0.0001)$ remained significant in our model. It was shown that patients with graduate degree of education compared to patients with high school diploma (odds ratio $=2.74,95 \%$ $\mathrm{CI}=1.14-6.58$ ) or middle school diploma (odds ratio $=8.22$, $95 \% \mathrm{CI}=1.43-47.36)$, and married patients compared to divorced patients (odds ratio $=0.31,95 \% \mathrm{CI}=0.13-0.78$ ) were less likely to suffer depressive symptoms. Also, the higher the number of hospitalization due to MS attacks $(\beta=1.10, p$ value $=0.02)$ and the greater the fear of COVID$19(\beta=1.07, p$ value $<0.0001)$, the greater was the chance to suffer depressive symptoms (Table 3 ).

In the present study sample, presence of anxious symptoms associated significantly with fear of COVID-19 ( $p$ value $\leq 0.0001)$, EDSS score $(p$ value $=0.004)$, and their employment status ( $p$ value $=0.045)$. It was revealed that patients with expression of anxious symptoms compared 
Table 3 Logistic regression for prediction of depressive symptoms in patients with multiple sclerosis

\begin{tabular}{llllr}
\hline Characteristics & $B$ & Beta & $95 \%$ of CI & $p$ value \\
\hline Age & -0.02 & 0.98 & $0.94-1.01$ & 0.273 \\
Gender & -0.12 & 0.88 & $0.44-1.78$ & 0.736 \\
History of psychological disease & 0.59 & 1.81 & $0.94-3.47$ & 0.073 \\
Occupational status & -0.15 & 0.85 & $0.48-1.53$ & 0.606 \\
Education & & & & 0.019 \\
$\quad$ Middle school degree & 2.10 & 8.22 & $1.43-47.36$ & \\
$\quad$ High school diploma & 1.00 & 2.74 & $1.14-6.58$ & \\
$\quad$ Associate/bachelor degree & 0.25 & 1.28 & $0.64-2.56$ & \\
$\quad$ Graduate degree & Reference & Reference & Reference & \\
Marital status & & & & 0.044 \\
$\quad$ Single & -0.93 & 0.39 & $0.15-1.00$ & \\
$\quad$ Married & -1.14 & 0.31 & $0.13-0.78$ & \\
$\quad$ Divorced & Reference & Reference & Reference & \\
Hospital admission & 0.10 & 1.10 & $1.01-1.20$ & 0.020 \\
Fear of COVID-19 & 0.07 & 1.07 & $1.03-1.12$ & $<0.0001$ \\
\hline
\end{tabular}

to other patients were more disable (EDSS score of 3.13 (1.5-4.5) vs 2.49 (1-3.5)), more afraid of COVID-19 (fear of COVID-19 score of 19.22 (15-24) vs 14.72 (10-18)), and more likely to be unemployed (66.1\% vs $54.1 \%$ ) (Table 4$)$.

In an enter model of univariate multiple binary logistic regression of anxiety, fear of COVID-19 ( $p$ value $<0.0001$ ) and EDSS score $(p$ value $=0.001)$ remained significant in predicting the prevalence of anxious symptoms. As shown in Table 5, patients with more severe fear of COVID-19 $(\beta=1.13)$ and more disability $(\beta=1.30)$ were more likely to express anxious symptoms (Table 5).

Entrance of Fear of COVID-19 Scale in our model did not change the significance of other factors neither for prediction of depression nor anxiety.

\section{Discussion}

The present cross-sectional study was conducted on 282 patients referred to the MS clinic of Sina Hospital in the summer of 2020 during the COVID-19 pandemic era to investigate the prevalence of presence of depressive and anxious symptoms in patients and their relationship with fear of COVID-19. In this sample, the prevalence of depressive symptoms in PWMS was $51.4 \%$, which was significantly associated with marital status, level of education, number of MS attacks leading to hospitalization, and the level of fear of COVID-19 in patients with MS. It was concluded that depressive symptoms were more prevalent in patients who have been separated from their partners, had more hospitalization due to MS attacks, were more afraid of COVID-19, and had lower level of education. Also, according to the BAI questionnaire, $60.6 \%$ of patients suffered anxious symptoms, but only $2.3 \%$ of them suffered from severe anxiety. It was shown that anxious symptoms prevalence was associated with the level of fear of COVID-19 and the severity of physical disability.

Recent studies with different questionnaires reported depression in up to $50 \%$ of patients with MS [15, 28, 29]; however, in one of the large-scale studies conducted in 2016 in Italy which similar to the present study made use of BDI questionnaire, depression prevalence was reported to be $33.9 \%$ [30]. Beside the type of questionnaire, sociodemographic characteristics of the study population and type of sampling would explain the difference between the reports, as Seyed Saadat et al.'s study in Iran revealed that $59.4 \%$ of patients with MS have been suffering from depression [31]. Furthermore, one of the most important biologic mechanisms developing depression in patients with MS is regional atrophy and lesion burden [15] leading to a higher prevalence of depression in patients with progressive courses [30]. Thereafter, including $23.9 \%$ of the sample with progressive types of the disease may justify this high prevalence.

Although the present study, in line with Ahorsu et al. [27], reported a significant relationship between fear of COVID19 and depression among patients with MS, but it cannot justify the higher prevalence of depression. Moreover, some recent studies [17, 32] comparing neuropsychological status of PWMS before the COVID-19 outbreak with its pandemic level did not report significant differences in this regard.

Solaro et al. [30] reported that PPMS patients were less likely to develop depression in their clinical course compared to RRMS or SPMS. However, the present study, in line with most other recent studies [31, 33, 34] including various demographic and clinical factors, did not find such a significant relationship. In our study population, only $22.5 \%$ of patients scored 4.5 or higher in EDSS and it did not show 
Table 4 Qualitative and quantitative variables across expression of anxious symptoms

\begin{tabular}{|c|c|c|c|}
\hline & \multicolumn{2}{|c|}{ Expression of anxious symptoms } & \multirow[t]{2}{*}{$p$ value } \\
\hline & Yes & No & \\
\hline Age & $35.92(32-40)$ & $35.6(29-41)$ & 0.354 \\
\hline Gender & & & 0.289 \\
\hline Male & $27(15.8 \%)$ & $23(20.7 \%)$ & \\
\hline Female & $144(84.2 \%)$ & $88(79.3 \%)$ & \\
\hline Education & & & 0.892 \\
\hline Middle school degree & $7(4.1 \%)$ & $3(2.7 \%)$ & \\
\hline High school diploma & $40(23.4 \%)$ & $24(21.6 \%)$ & \\
\hline Associate/bachelor degree & $88(51.5 \%)$ & $61(55.0 \%)$ & \\
\hline Graduate degree & $36(21.1 \%)$ & $23(20.7 \%)$ & \\
\hline Occupational status & & & 0.045 \\
\hline Employed & $58(33.9 \%)$ & $50(45.9 \%)$ & \\
\hline Unemployed & $113(66.1 \%)$ & $59(54.1 \%)$ & \\
\hline Marital status & & & 0.116 \\
\hline Single & $49(28.7 \%)$ & $44(39.6 \%)$ & \\
\hline Married & $98(57.3 \%)$ & $57(51.4 \%)$ & \\
\hline Divorced & $24(14 \%)$ & $10(9 \%)$ & \\
\hline MS type & & & 0.089 \\
\hline CIS & $8(4.7 \%)$ & $11(9.9 \%)$ & \\
\hline RRMS & $117(68.4 \%)$ & $78(70.3 \%)$ & \\
\hline PPMS & $12(7.0 \%)$ & $10(9.0 \%)$ & \\
\hline SPMS & $34(19.9 \%)$ & $12(10.8 \%)$ & \\
\hline Disease duration & $7.49(3-11)$ & $7.98(3.5-10)$ & 0.487 \\
\hline Treatment duration & $6.39(3-9)$ & $5.95(3-8)$ & 0.364 \\
\hline Hospital admission due to MS attacks & $2.34(0-3)$ & $2.05(0-2)$ & 0.251 \\
\hline EDSS & $3.13(1.5-4.5)$ & $2.49(1-3.5)$ & 0.004 \\
\hline Drug & & & 0.139 \\
\hline Immunomodulatory & $91(53.2 \%)$ & $69(62.2 \%)$ & \\
\hline Immunosuppressive & $80(46.8 \%)$ & $42(37.8 \%)$ & \\
\hline History of psychological disease & & & $\mathbf{0 . 7 7 0}$ \\
\hline Yes & $33(19.3 \%)$ & $23(20.7 \%)$ & \\
\hline No & $138(80.7 \%)$ & $88(79.3 \%)$ & \\
\hline COVID-19 infection & & & 0.457 \\
\hline Yes & $14(8.2 \%)$ & $12(10.8 \%)$ & \\
\hline No & $157(91.8 \%)$ & $99(89.2 \%)$ & \\
\hline Fear of COVID-19 & $19.22(15-24)$ & $14.72(10-18)$ & $\leq \mathbf{0 . 0 0 0 1}$ \\
\hline
\end{tabular}

$C I S$, clinically isolated syndrome; RRMS, relapsing-remitting multiple sclerosis; $P P M S$, primary progressive multiple sclerosis; SPMS, secondary progressive multiple sclerosis; EDSS, expanded disability status scale; $B D I$, Beck depression inventory; $B A I$, Beck anxiety inventory. any differences regarding the prevalence of depression. On the other hand, other recent studies [33, 34] have shown that patients with more severe disability may more likely experience depression. However, considering the number of attacks along with other demographic and clinical factors, Seyed Saadat et al. [31] omitted the effect of EDSS. Therefore, future studies with larger sample size are required to reevaluate the EDSS and disease course on depressive symptoms in patients with MS.

Regarding anxiety in patients with MS, according to Beck questionnaire, $79.1 \%$ of patients scored less than 21 (mild anxiety), $17.7 \%$ of patients scored 22 to 35 (moderate anxiety), and only $3.2 \%$ of patients scored more than 36 (severe anxiety). In this regard, according to the different types of questionnaires that have been filled out by patients in different periods of sociopolitical events all over the world, prevalence of anxiety is very heterogeneous. For example, during the COVID-19 outbreak in Italy, Capuano et al. [32] found the prevalence of anxiety in patients with MS at $16.4 \%$, according to the State-Trait Anxiety Inventory (STAI). In another study conducted by Da Silva et al. [35] in 2011 in Portugal on MS patients with the Hospital Anxiety 
Table 5 Logistic regression for prediction of anxious symptoms in patients with multiple sclerosis

\begin{tabular}{lllcc}
\hline Characteristics & $B$ & Beta & $95 \%$ of CI & $p$ value \\
\hline Age & -0.00 & 0.99 & $0.96-1.03$ & 0.82 \\
Gender & 0.19 & 1.21 & $0.60-2.45$ & 0.58 \\
$\begin{array}{l}\text { History of psycho- } \\
\quad \text { logical disease }\end{array}$ & -0.25 & 0.77 & $0.40-1.50$ & 0.45 \\
Occupational status & -0.22 & 0.79 & $0.45-1.39$ & 0.42 \\
EDSS & 0.26 & 1.30 & $1.11-1.53$ & 0.001 \\
Fear of COVID-19 & 0.12 & 1.13 & $1.08-1.19$ & $<0.0001$ \\
\hline
\end{tabular}

EDSS, expanded disability status scale

and Depression Scale (HADS), anxiety level was estimated to be $51 \%$ and $26.6 \%$, respectively. A recent review article on psychological disease in PWMS estimated the prevalence of anxiety at $22.1 \pm 8.9 \%$ [16].

Recent studies have reported different results of new prevalence of anxiety during the pandemic era. For instance, Stojanov et al. [17] reported an increase in the rate of anxiety in patients with MS, while Capuano et al. [32] did not find any changes. However, it depends on the time of study (early stages vs peak stages) and the type of anxiety questionnaire, but the association between fear of COVID-19 and anxiety has been reported [27].

In line with Mazza et al. [34] and Pham et al. [35], neither occupational nor marital status has an effect on the prevalence of anxiety in patients with MS. However, contrary to Pham et al. [35], in the present study, EDSS score in pandemic period was significantly associated with anxiety prevalence in patients with MS.

The most important limitation in the present study hides in the data gathering stage where information is obtained from patients subjectively. Although this method is acceptable for demographic features and questionnaires of anxiety, depression, and fear of COVID-19, but it will be erroneous in receiving clinical factors from patients. Furthermore, according to DSM V criteria, a close and specific interview with patients rather than BDI-II and BAI questionnaires needs to be done for diagnosis of depression and anxiety. On the other hand, some relatively common conditions like cognitive impairment in PWMS can both interfere with understanding of questionnaire and be confused with depressive symptoms itself. Long aside EDSS, which can reflect the neurological damage, different personalities percept their condition differently, and wellbeing perception could result in better emotional status. This study lacks of control group for comparing psychological consequences of PWMS with healthy individuals. Another important limitation was the method of crosssectional study and also the lack of information about the psychiatric status of patients before the pandemic. Future studies are suggested to examine longitudinal psychiatric changes in patients to determine the effect of COVID19 pandemic on prevalence of anxiety and depression in PWMS with more certainty.

In conclusion, beyond the physical morbidity and mortality of the COVID-19 pandemic among patients with chronic diseases, it has some concealed effects leading to more exacerbations and reducing patients' performance [20]. This study reported fear of COVID-19 as an important significant factor in predicting the prevalence of depression and anxiety in PWMS. Future studies can find the other consequences of fear of COVID-19 on patients' life, including their medication seek and treatment adherence.

Author contribution Mohammad Alirezaei: investigation, resources, software, formal analysis, writing-original draft. Sharareh Eskandarieh: investigation, resources. Mohammad Ali Sahraian: investigation, resources. Abdorreza Naser Moghadasi: conceptualization, investigation, resources, writing-review and editing, supervision.

Funding The author(s) disclosed receipt of the following financial support for the research, authorship, and/or publication of this article: This work was supported by Tehran University of Medical Sciences.

Data availability The data that support the findings of this study are available on request from the first and corresponding author. The data are not publicly available due to information that could compromise the privacy of research participants.

Code availability All data were analyzed via SPSS version 22.

\section{Declarations}

Conflict of interest The authors declare no competing interests.

Ethics approval This study was performed in line with the principles of the Declaration of Helsinki. Approval was granted by the Ethics Committee of Tehran University of Medical Sciences (IR.TUMS. SINAHOSPITAL.REC.1399.065).

Consent to participate Prior to administration the online survey, a paragraph about the study conduction and aim was handed out among participants and if they accepted the terms, they completed the survey.

Consent for publication Patients accepted publishing their clinical data prior to filling the survey.

\section{References}

1. Shi Y, Wang G, Cai XP, Deng JW, Zheng L, Zhu HH, Zheng M, Yang B, Chen Z (2020) An overview of COVID-19. J Zhejiang Univ Sci B 21(5):343-360. https://doi.org/10.1631/jzus.B2000083

2. Zhu N, Zhang D, Wang W, Li X, Yang B, Song J et al (2020) A novel coronavirus from patients with pneumonia in China, 2019. N Engl J Med 382(8):727-733 
3. Shatla MM, Khafagy AA, Bulkhi AA, Aljahdali IA (2020) Public concerns and mental health changes related to the COVID-19 pandemic lockdown in Saudi Arabia. Clin Lab 66(10). https://doi. org/10.7754/Clin.Lab.2020.200614

4. Kessler RC, Gruber M, Hettema JM, Hwang I, Sampson N, Yonkers KA (2008) Co-morbid major depression and generalized anxiety disorders in the National Comorbidity Survey follow-up. Psychol Med 38(3):365-374

5. Kessler RC, Berglund P, Demler O, Jin R, Merikangas KR, Walters EE (2005) Lifetime prevalence and age-of-onset distributions of DSM-IV disorders in the National Comorbidity Survey replication. Arch Gen Psychiatry 62(6):593-602

6. Wittchen HU, Zhao S, Kessler RC, Eaton WW (1994) DSM-III-R generalized anxiety disorder in the National Comorbidity Survey. Arch Gen Psychiatry 51(5):355-364

7. Pietrzak RH, Kinley J, Afifi TO, Enns MW, Fawcett J, Sareen J (2013) Subsyndromal depression in the United States: prevalence, course, and risk for incident psychiatric outcomes. Psychol Med 43(7):1401-1414

8. Kessler RC, Zhao S, Blazer DG, Swartz M (1997) Prevalence, correlates, and course of minor depression and major depression in the National Comorbidity Survey. J Affect Disord 45(1-2):19-30

9. Lewinsohn PM, Klein DN, Durbin EC, Seeley JR, Rohde P (2003) Family study of subthreshold depressive symptoms: risk factor for MDD? J Affect Disord 77(2):149-157

10. Kessler RC, Ormel J, Petukhova M, McLaughlin KA, Green JG, Russo LJ et al (2011) Development of lifetime comorbidity in the World Health Organization world mental health surveys. Arch Gen Psychiatry 68(1):90-100

11. Torales J, O’Higgins M, Castaldelli-Maia JM, Ventriglio A (2020) The outbreak of COVID-19 coronavirus and its impact on global mental health. Int J Soc Psychiatry 66(4):317-320

12. Zhang Y, Ma ZF (2020) Impact of the COVID-19 pandemic on mental health and quality of life among local residents in Liaoning Province, China: a cross-sectional study. Int J Environ Res Public Health 17(7)

13. Maric GD, Pekmezovic TD, Mesaros ST, Tamas OS, Ivanovic JB, Martinovic VN et al (2020) The prevalence of comorbidities in patients with multiple sclerosis: population-based registry data. Neurol Sci

14. Patten SB, Marrie RA, Carta MG (2017) Depression in multiple sclerosis. Int Rev Psychiatry 29(5):463-472

15. Boeschoten RE, Braamse AMJ, Beekman ATF, Cuijpers P, van Oppen P, Dekker J et al (2017) Prevalence of depression and anxiety in multiple sclerosis: a systematic review and meta-analysis. J Neurol Sci 372:331-341

16. Stojanov A, Malobabic M, Milosevic V, Stojanov J, Vojinovic S, Stanojevic G et al (2020) Psychological status of patients with relapsing-remitting multiple sclerosis during coronavirus disease-2019 outbreak. Mult Scler Relat Disord 45:102407

17 Naser Moghadasi A (2020) One aspect of coronavirus disease (COVID-19) outbreak in Iran: high anxiety among MS patients. Mult Scler Relat Disord 41:102138

18. Pashazadeh Kan F, Hoseinipalangi Z, Ahmadi N, Hosseinifard H, Dehnad A, Sadat Hoseini B et al (2020) Global, regional and national quality of life in patients with multiple sclerosis: a global systematic review and meta-analysis. BMJ Support Palliat Care

19. Artemiadis AK, Anagnostouli MC, Alexopoulos EC (2011) Stress as a risk factor for multiple sclerosis onset or relapse: a systematic review. Neuroepidemiology 36(2):109-120

20. Arnett PA, Barwick FH, Beeney JE (2008) Depression in multiple sclerosis: review and theoretical proposal. J Int Neuropsychol Soc $14: 691-724$
21. Pakpour AH, Griffiths MD (2020) The fear of COVID-19 and its role in preventive behaviors. J Concurr Disord ISSN 2562-7546

22. Satici B, Gocet-Tekin E, Deniz ME, Satici SA (2020) Adaptation of the Fear of COVID-19 Scale: its association with psychological distress and life satisfaction in Turkey. Int J Ment Health Addict $1-9$

23. Sacco R, Santangelo G, Stamenova S, Bisecco A, Bonavita S, Lavorgna L et al (2016) Psychometric properties and validity of Beck depression inventory II in multiple sclerosis. Eur J Neurol 23(4):744-750

24. Osman A, Hoffman J, Barrios FX, Kopper BA, Breitenstein JL, Hahn SK (2002) Factor structure, reliability, and validity of the Beck anxiety inventory in adolescent psychiatric inpatients. J Clin Psychol 58(4):443-456. https://doi.org/10.1002/jclp.1154

25. Ahorsu DK, Lin CY, Imani V, Saffari M, Griffiths MD, Pakpour AH (2020) The Fear of COVID-19 Scale: development and initial validation. Int J Ment Health Addict 1-9

26. Amtmann D, Kim J, Chung H, Bamer AM, Askew RL, Wu S et al (2014) Comparing CESD-10, PHQ-9, and PROMIS depression instruments in individuals with multiple sclerosis. Rehabil Psychol 59(2):220-229

27. Schippling S, O’Connor P, Knappertz V, Pohl C, Bogumil T, Suarez $G$ et al (2016) Incidence and course of depression in multiple sclerosis in the multinational BEYOND trial. J Neurol 263(7):1418-1426

28. Solaro C, Trabucco E, Signori A, Martinelli V, Radaelli M, Centonze D et al (2016) Depressive symptoms correlate with disability and disease course in multiple sclerosis patients: an Italian multi-center study using the Beck depression inventory. PLoS One 11(9):e0160261

29. SeyedSaadat SM, Hosseininezhad M, Bakhshayesh B, SeyedSaadat SN, Nabizadeh SP (2014) Prevalence and predictors of depression in Iranian patients with multiple sclerosis: a population-based study. Neurol Sci 35(5):735-740

30. Capuano R, Altieri M, Bisecco A, d'Ambrosio A, Docimo R, Buonanno D et al (2020) Psychological consequences of COVID-19 pandemic in Italian MS patients: signs of resilience? J Neurol

31. Bamer AM, Cetin K, Johnson KL, Gibbons LE, Ehde DM (2008) Validation study of prevalence and correlates of depressive symptomatology in multiple sclerosis. Gen Hosp Psychiatry 30(4):311-317

32. Chwastiak L, Ehde DM, Gibbons LE, Sullivan M, Bowen JD, Kraft GH (2002) Depressive symptoms and severity of illness in multiple sclerosis: epidemiologic study of a large community sample. Am J Psychiatry 159(11):1862-1868

33. da Silva AM, Vilhena E, Lopes A, Santos E, Goncalves MA, Pinto $\mathrm{C}$ et al (2011) Depression and anxiety in a Portuguese MS population: associations with physical disability and severity of disease. J Neurol Sci 306(1-2):66-70

34. Mazza C, Ricci E, Biondi S, Colasanti M, Ferracuti S, Napoli C et al (2020) A nationwide survey of psychological distress among Italian people during the COVID-19 pandemic: immediate psychological responses and associated factors. Int J Environ Res Public Health 17(9)

35. Pham T, Jette N, Bulloch AGM, Burton JM, Wiebe S, Patten SB (2018) The prevalence of anxiety and associated factors in persons with multiple sclerosis. Mult Scler Relat Disord 19:35-39

Publisher's Note Springer Nature remains neutral with regard to jurisdictional claims in published maps and institutional affiliations. 